\title{
Research on Intelligent Model of Transaction Platform in Rural E-Commerce Based on Big Data Analysis
}

\author{
Hongsheng $\mathrm{Xu}^{1, \mathrm{a}}$ and Ganglong Fan ${ }^{1}$ \\ ${ }^{1}$ Luoyang Normal University, Luoyang, 471934, China \\ axhs_Is@sina.com
}

Keywords: E-commerce; Big data; Rural e-commerce trading platform; Data mining; Intelligent

\begin{abstract}
This paper first discuss the large scale, multi modal and growth of the data, and analyze the application of intelligent data mining in the aspects of computing, effectiveness and high efficiency. This paper analyzes the current situation of development of e-commerce in rural areas, as well as the purpose and significance of building a smart rural e-commerce trading platform. The paper presents intelligent model of transaction platform in rural e-commerce based on big data analysis. Finally, the experimental results show that the proposed method is effective.
\end{abstract}

\section{Introduction}

Big data analysis is not in a certain period of time with conventional machines and software and hardware for their perception, access, and management, processing and service data collection. IBM will be the characteristics of large data summed up to three $\mathrm{V}$, that is, the large quantization (Volume), (Variety) and fast (Velocity) that is, the resulting large data capacity.

Big data of large-scale, multi modality and the rapid growth of big data to mining challenge is in many aspects and levels [1]. Derived from the problem has both related to the field, but also has interdisciplinary. Therefore, it is necessary on the basis of the existing research results, new theories and new methods for the new perspective of the development of data mining, promote the development and application of the data subject.

With the transformation of the traditional agriculture to the modern agriculture, the electronic commerce development environment is becoming mature, and the development of e-commerce will help to drive the overall development of the agricultural industry in Henan. Henan agricultural sites although the number of many, but most agricultural website only in the primary stage of the application of e-commerce, mainly for the issuance of agricultural information, do not offer or provide only limited trading services, lack of product recommendation, price comparison, traders of credit evaluation, real-time market monitoring, product traceability traceability and other service functions.

The formation process of rural e-commerce data: rural e-commerce data is accompanied by consumers behavior, conform to the needs of the consumers, rural comprehensive e-commerce are widely distributed in e-commerce platform and active in various social media and enterprise internal system. As the types of e-commerce data and data are very diverse, the content of the data includes the consumer's comments, consumer behavior information and the consumer's social information, etc. The paper presents intelligent model of transaction platform in rural e-commerce based on big data analysis.

\section{Intelligent Big Data Analysis and Data Mining Process}

Data in the modern information society, data resource subject status has become the consensus of the academic circles and the business community [2]. Possessed the foreseeable important role in promoting, big data has entered the strategic research plan for the world's major economies, will vigorously promote the collection of data, access, organization and the development. From the data for data mining and knowledge discovery is one of the strategic problems of big data applications. 
Some common methods of feature extraction, data mining technology is a attribute reduction process, the actual transformation properties, attributes or characteristics of the transform is linear combinations of original attributes set, appear smaller and more refined a set of attributes. Common algorithms such as principal component analysis, it is factor analysis and non negative matrix factorization.

MapReduce is in large-scale unstructured data management level for people to provide a parallel processing architecture. And experience in data analysis and data mining level can be computationally challenging, although there have been some work, but also in the use the MapReduce data pipe stage, has not entered for data mining tasks, the following formula 1 below.

$$
d_{i}^{1}(t)=\frac{x_{i}^{1}(t)-x_{i+1}^{1}(t+1)}{x_{i}^{1}(t)}
$$

The main sources of data such as: E-mail, search engine search, pictures, audio, video, social networking sites, WeChat micro-blog, a variety of applications and app e-commerce and electronic communications, etc. In real life, the number and type of e-commerce shopping platform is more and more, the typical Facebook data of social networking sites is amazing, PB measurement is not enough. Data storage unit is not only GB, MB, etc.

Big data is referred to in a certain period of time with conventional software tools to its content to crawl, management and processing of the data set [3]. Therefore, data mining must first solve the problem of big data can be considered ", which requires the reasonable separation of big data, is a large data set of granulation, then the parallel processing strategy, MapReduce is based on this strategy in data management and practical results.

Because of the universality and universality of data resources, the characteristics, functions and properties of the related network data have attracted more and more attention. For example, artificial neural network related data information includes many kinds of data information, by data mining technology to analyze the application, with the help of computer, realized for the complex pattern of trend analysis; in addition, genetic algorithm in evaluating other algorithms suitable should be used very widely, based on the principle of biological evolution, assumptions, the virtual information data growth process, to construct a virtual and real information resources, as shown in equation 2 [4].

$$
w_{i+1}^{1}(t+1)=\left(1-w d_{i}^{1}(t)\right) x_{i}^{1}(t)-r s_{i} \alpha N^{1}(t)
$$

Granulation of data is the data decomposition, or the decomposition of the local data according to the requirement analysis of the re organization. It may be in accordance with the data of some of the natural attributes of simple decomposition may also be according to problem solving based on frameworks, theories, methods and techniques of local data content requirements for data decomposition and organization [5]. Granulation is based on granular computing framework to solve complex problems based in equation (3). The scale of the data is mainly reflected in two aspects of sample size of mass characteristics and scale of high dimensional.

$$
|f(c)|=M=\max _{0 \leq x \leq \frac{1}{2 A}}|f(x)|
$$

Linear complexity clustering method for large data blocks [6]. And in the process of testing, for each test sample, first identify and tested sample clusters where the nearest cluster center distance as the new training sample set, then the new training set classification model to classify, because of the cluster after cluster sample of high similarity, so the algorithm can achieve both to reduce the amount of computation, and can keep high classification accuracy.

Step1: Reverse index need to rely on the Cassandra database implementation, the first system to submit the write action, recorded in the log;

Step2: To find out the projection matrix $\mathrm{Z}$ in the landmark $\mathrm{X}$, where $\mathrm{j} u$ is the $\mathrm{j}$ matrix $\mathrm{U}$ column, $\mathrm{U}$ $\mathrm{I}$ is a namely the above $\mathrm{r} \mathrm{Xi}$ recently; 
Step3: Document oriented NoSQL database in addition to the requirements of large data storage, query performance is also a higher requirement;

Step4: According to the matrix Z, the calculation of the TZZ before K feature vector A, as shown below:

$$
\lambda p_{n}=n \mu p_{n+1}, p_{n+1}=\frac{\lambda}{n \mu} p_{n}=\frac{\rho^{n+1}}{n \cdot n !} p_{0}
$$

Step5: Using LSC clustering method to cluster the training samples, get the M cluster center C1, $\mathrm{C} 2, \mathrm{C} 3, \ldots, \mathrm{Cm}$;

Step6: In accordance with the above method, if the original cluster is added to a node, the key data of the adjacent nodes of the node must be migrated to the new node;

Step7: If $k(n>100)$ then there will be a lot of false points in the big data classification;

Step8: The FCA and rough sets are used to carry out the final clustering of the feature vector B, and the $\mathrm{K}$ sub clusters are output.

Therefore, the process of large data processing is very complex. Because big data has the characteristics of strong timeliness, so it can quickly generate data, so it is more need for high-speed data storage, and then the data for real-time and effective management. Especially in the management of large data, there are still a large amount of data content is not consistent with the information content, it is difficult to improve the data value, so the data of model innovation has important significance.

\section{Analysis on Present Situation of Electronic Commerce Transaction Platform in Rural Areas}

Agriculture is the basic industry of our country, and it plays a supporting role in the economic and social development of our country. However, the agricultural product has a strong seasonal, scattered spatial distribution [7]. At the same time, there are is not easy to preserve, more circulation cost high, the agricultural products of the small-scale peasant economy and production, unified market has a sharp contradiction.

Mobile Internet applications and the development of wireless data communication technology, and it is to provide a solid foundation for the development of mobile e-commerce. But the traditional mobile e-commerce development and application of high cost, long cycle and need the corresponding client program development for different types of mobile phone operating system, agricultural product electronic commerce public service platform of mobile e-commerce system using HTML5, CSS3, jQuery technology, to let the user through a browser to access web application, mobile e-commerce transactions [8].

According to the specific needs of the consumer market and the actual situation of enterprises, for the design to meet the business requirements of the agricultural e-commerce platform, the e-commerce system to complete the procurement of goods, storage of goods, sales of goods, goods distribution to finally receive the payment of the entire business process, and realize the information query, the release of supply and demand, online ordering etc.. The subject set up a number of functional modules: portal content management; business management; shop management; supply and demand information management; shopping management; commodity intelligent search engine; customer relationship management, etc..

Rural e-commerce trading platform is the wholesale market for agricultural products based on agricultural trading market, financial and material integrated management using information technology to improve the market's own work quality and efficiency, promote the standardization of market management, the establishment of e-commerce transaction sub platform, order management, customer management, goods management other functions, at the same time, using the supply chain management module, interact through the cloud data center and the organization and management of logistics center, logistics organization and management; through a unified access interface, through the Internet, the use of mobile phone, tablet computer, notebook and other computing devices to interact with customers, farmers and other types of users. 


\section{Experiments and Analysis}

In recent years, with the increasing development of mobile devices, the impact of mobile devices on e-commerce is becoming more and larger. As the mobile terminal has many advantages, easy to move, and very fast characteristics, to promote the rapid development of e-commerce, through the development of a large number of data generated [9]. Therefore, the electronic commerce should be further excavated, to re create a new value, to improve the competitiveness of e-commerce.

In the construction of the whole system is on the basis of existing agricultural e-commerce platform, through the use of advanced and feasible means of modern technology and management mode, construction a major agricultural products wholesale market comprehensive electronic trading platform for system design idea, system architecture and the technology selection of the platform are required to have certain advanced, forward-looking, consider the growth of business in a certain period of time:

(1) The comprehensive and wide application of big data technology has brought new opportunities for innovation [10]. Especially under the background of the era of big data, the value of the total extraction of large data, and the data used in e-commerce in the various processes and links, the formation of a new business model, as is shown by equation(5).

$$
M(x, y) d x+N(x, y) d y=0, \exists \mu(x, y) \neq 0
$$

(2) Based on Java EE three-tier architecture "layer and middle layer - data layer development technology by using large data model of mobile e-commerce system, presentation layer using HTML5, CSS3, jQuery technology, eliminate the differences between different platforms, truly cross platform.

(3) The electronic commerce transaction platform of agricultural products adopts the mature browser /Web server / database server (B/W/S) architecture, the whole system is advanced and adaptable, and can meet the needs of the future.

In intelligent data analysis, build rural electronic commerce is by identifying the needs in different stages of the consumer, the rural consumer demand design and develop of the personalized products on the basis of, for the benefit of urban and rural consumers actively participate in this link to be in. According to the needs of consumers to customized products, has many characteristics: make full use of the advantages of network resources, extensive use of various social networking sites, through the platform of e-commerce in rural areas, there are a variety of mobile terminal channels, to fully received a cancellation fee of information.

In order to verify the effectiveness of the algorithm, this paper is in the win7 system under the MATLAB software to carry out the experiment. In this paper, the classification accuracy and time as the evaluation index, the random block FCA algorithm, LSC algorithm based on KNN algorithm for comparison, the experimental data from the LIBSVM and UCI data sets, as is shown by Fig. 1.

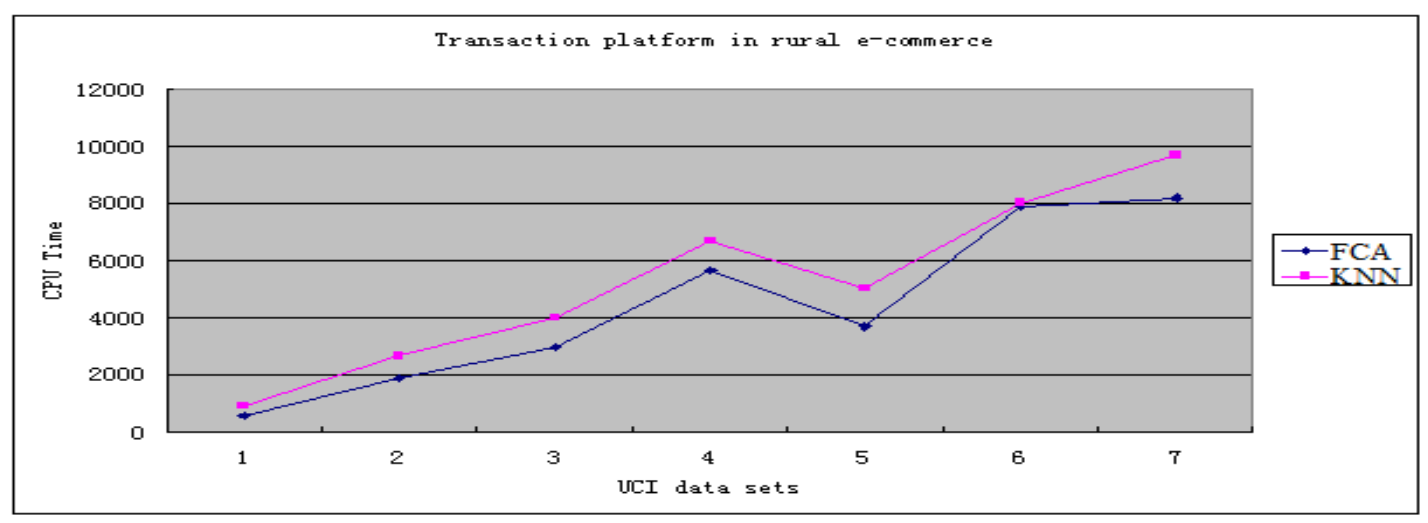

Figure 1. Intelligent model of transaction platform in rural e-commerce based on big data analysis

Therefore, through the above experiment shows that using FCA based clustering algorithm in classification accuracy is higher, greatly mention speed, so that it can be applied in a large rural electronic commerce data. 


\section{Summary}

The paper presents intelligent model of transaction platform in rural e-commerce based on big data analysis. With big data background as the main starting point, a comprehensive analysis of rural e-commerce enterprises in the era of big data, all kinds of opportunities and challenges faced by. Especially big data as a factor of production, caused great attention in rural enterprises, and these enterprises will be the big data as a very important strategic resource.

\section{Acknowledgements}

This paper is supported by the science and technology research major project of Henan province Education Department (13B520155) and Henan Province basic and frontier technology research project (142300410303).

\section{References}

[1] Hyoung woo Park, Il Yeon Yeo, Haengjin Jang, Seo-Young Noh, "Simulation based Analysis on Big Data Service Bottleneck for Data Center", JNIT, Vol. 4, No. 8, pp. 185 189, 2013

[2] Wei Li, Hongtu Zhang, Tingting An, "Optimal Decision-Making in E-Commerce Platform Based On Optimal Stopping Theory", JCIT, Vol. 8, No. 8, pp. $922 \sim 929,2013$.

[3] Sirivimol Thanchalatudom, Namfon Assawamekin, "Using Big Data Technology for Information Management in Hybrid Learning System", RNIS, Volume 12, pp. 179 182, 2013.

[4] JinHui Lei, XianFeng Yang, "Construction the E-commerce Trading Platform Based on Rough Set Data Mining Technology", JCIT, Vol. 8, No. 3, pp. 460 469, 2013.

[5] Hyoung Woo Park, Heejun Yoon, Haengjin Jang, Jongsuk Ruth Lee, "Study on the Automatic Alteration of the Transmission Style for Big Data Transfer according to the Change of Internet Status", JNIT, Vol. 4, No. 9, pp. 89 95, 2013.

[6] Dung-Hai Liang, Dong-Shong Liang, Min-Jer Lu, James K.C. Chen, "Integration and Innovation Management Applications on Urban and Rural Planning", IJACT, Vol. 4, No. 14, pp. 233 241, 2012.

[7] Dawei Sun, Ge Fu, Xinran Liu, Hong Zhang, "Optimizing Data Stream Graph for Big Data Stream Computing in Cloud Datacenter Environments", IJACT, Vol. 6, No. 5, pp. $53 \sim$ 65, 2014.

[8] Sun Chengshuang, Shen Liyin, Gan Lin, "An Analysis on Competitiveness Enhancement by Applying E-commerce to Construction Corporations", JDCTA, Vol. 7, No. 8, pp. $772 \sim 780$, 2013.

[9] Fan Yang, "The Research of E-Commerce Recommendation System Based on Cloud Computing", IJACT, Vol. 4, No. 16, pp. 256 263, 2012.

[10] Yiqun Li, Cong Shu, Li Xiong, "Information Services Platform of International Trade Based on E-commerce ", AISS, Vol. 3, No. 1, pp. 78 86, 2011. 